\title{
Beauty jet production at HERA with ZEUS
}

\author{
Mykhailo LISOVYI ${ }^{* \dagger}$ \\ DESY and University of Hamburg \\ E-mail: mlisovyi@mail.desy.de
}

Two recent measurements of beauty production in deep inelastic ep scattering with the ZEUS detector are presented. In the first analysis, beauty production was identified using muons associated to a jet. In the second measurement, beauty content was extracted using the decay length significance and invariant mass of secondary vertices. Measured differential cross sections were compared to theoretical predictions. The beauty contribution to the proton structure function $F_{2}$ was extracted.

XXIst International Europhysics Conference on High Energy Physics

21-27 July 2011

Grenoble, Rhône-Alpes France

\footnotetext{
* Speaker.

${ }^{\dagger}$ on behalf of the ZEUS Collaboration
} 


\section{Introduction}

Beauty production in deep inelastic scattering (DIS) regime is important for several reasons. Production of beauty quarks is dominated by the boson-gluon fusion process, hence this process provides a direct probe of the gluon component of the proton. In addition, the mass of $b$ quarks provides a large scale for perturbative QCD (pQCD) calculations, but on the other hand, presence of multiple hard scales (photon virtuality, quark mass and momentum) can spoil the series convergence due to large logarithmic terms. Therefore, a precise measurement of beauty production allows to distinguish between different theoretical approaches to treat mass effects in pQCD predictions.

\section{Measurement of beauty production using events with a jet and a muon}

The production of $b$ quarks was measured in events with a jet and a muon with the ZEUS detector at HERA using $114 \mathrm{pb}^{-1}$ of data [1]. The reaction $e p \rightarrow e b \bar{b} X \rightarrow e$ jet $\mu X$ was measured in the kinematic region of $Q^{2}>2 \mathrm{GeV}^{2}, 0.05<y<0.7, E_{\mathrm{T}}^{\text {jet }}>5 \mathrm{GeV}$ and $-2.0<\eta^{\text {jet }}<2.5$ including a muon of $p_{\mathrm{T}}^{\mu}>1.5 \mathrm{GeV}$ and $-1.6<\eta^{\mu}<2.5$ inside a cone of $\Delta R<0.7$ to the jet axis, where $\Delta R=\sqrt{(\Delta \phi)^{2}+(\Delta \eta)^{2}}$. Jets were reconstructed using the $k_{\mathrm{T}}$ cluster algorithm in the massive mode with the $E_{\mathrm{T}}-$ recombination scheme.

The beauty signal was extracted from the distribution of the transverse momentum of the muon with respect to the momentum of the associated jet, $p_{\mathrm{T}}^{\text {rel }}$. The fraction of beauty events in the data was obtained from a two-component fit to the shape of the measured $p_{\mathrm{T}}^{\text {rel }}$ distribution (see Fig. 11).

Figure 2 shows the measured differential cross section as a function of the photon virtuality compared to the next-to-leading-order (NLO) QCD calculations as well as the MC predictions scaled to the data. Both predictions describe the shape of the measured cross sections reasonably well.

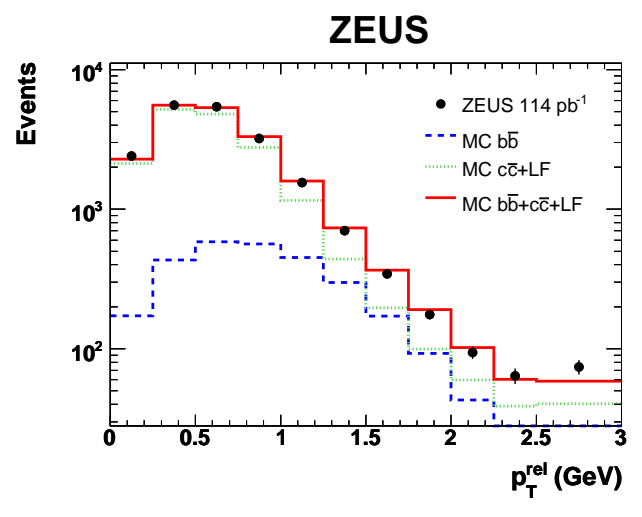

Figure 1: Measured $p_{\mathrm{T}}^{\text {rel }}$ distribution and the fit using templates obtained from the Monte Carlo simulations for beauty, charm and light flavour (LF) events.

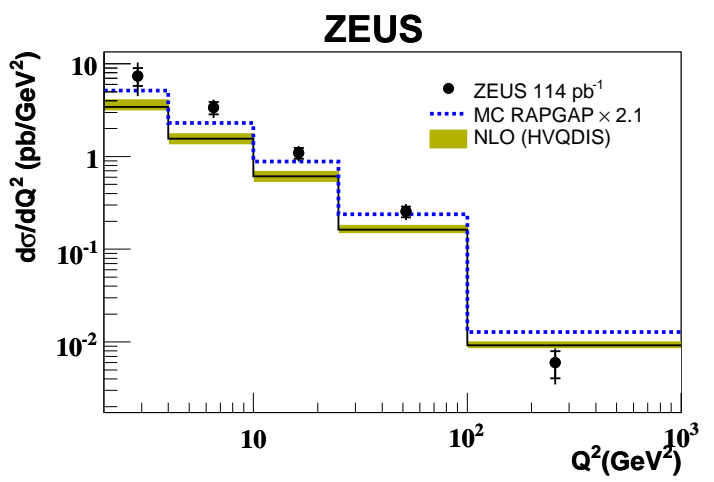

Figure 2: Differential beauty cross sections as a function of $Q^{2}$ compared to the Monte Carlo and the NLO QCD predictions. 


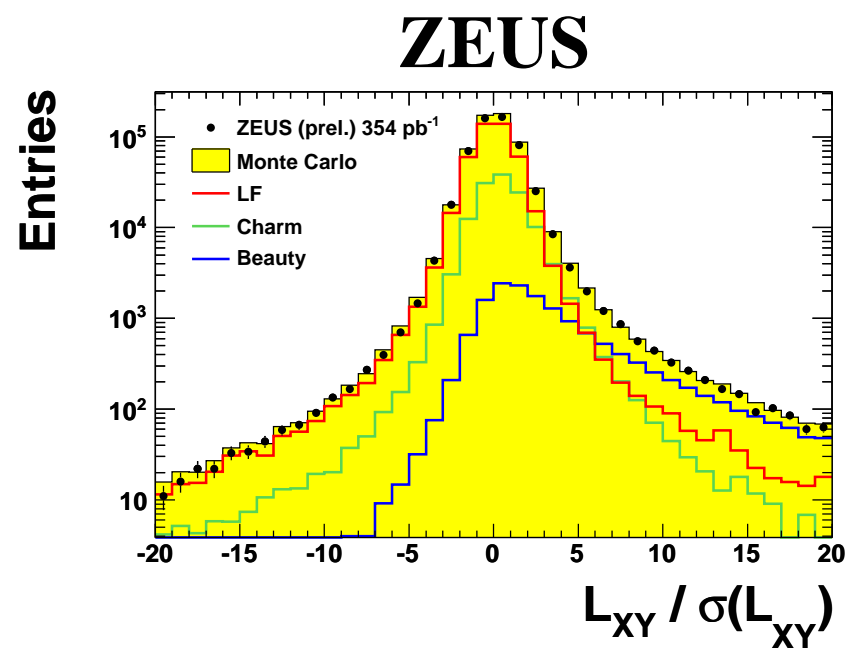

Figure 3: Decay length significance for the data and the Monte Carlo samples for different flavour production in the region $2<M_{V T X}<6 \mathrm{GeV}$.

\section{Measurement of beauty production using inclusive secondary vertices}

A measurement of beauty quark production in DIS was also done using the decay length significance and invariant mass of secondary vertices, $M_{V T X}$, using an integrated luminosity of $354 \mathrm{pb}^{-1}$ collected between 2004 and 2007 [2]. The kinematic region is defined by: $5<Q^{2}<1000 \mathrm{GeV}^{2}$, $0.02<y<0.7, E_{\mathrm{T}}^{\text {jet }}>5 \mathrm{GeV}$ and $-1.6<\eta^{\text {jet }}<2.2$. The same clustering algorithm as described in Section 2 was used to reconstruct jets. A secondary vertex was fitted for any selected jet if at least two tracks with $p_{\mathrm{T}}>500 \mathrm{MeV}$ were associated to the jet using $\Delta R<1$ criteria.

The beauty fraction in the sample was determined using the decay length significance, $S=$ $L_{X Y} / \sigma\left(L_{X Y}\right)$, where $L_{X Y}$ is the decay length projected on the jet axis in the plane perpendicular to the beam (the $X Y$ plane) and $\sigma\left(L_{X Y}\right)$ is its uncertainty. The significance distribution observed in the data as well as beauty, charm and LF distributions predicted by the MC simulations are shown in Fig. 3. The left side of the $S$ distribution was mirrored on and subtracted from the positive side to suppress the symmetric LF component. To extract the beauty contribution in the data a binned $\chi^{2}$ fit of the mirrored significance distributions simultaneously in three bins of $M_{V T X}$ was performed.

Figure 4 shows the measured differential cross sections for beauty jet production as a function of $E_{\mathrm{T}}^{\text {jet }}$ and $\eta^{\text {jet }}$. NLO QCD predictions as well as Monte Carlo simulations scaled to the data are also shown. The shapes of the measured cross sections are described by both predictions. The data tend to lie $20-30 \%$ above the NLO QCD calculations.

\section{Extraction of the beauty contribution to the proton structure function $F_{2}, F_{2}^{b \bar{b}}$}

In both analyses presented in Section 2 and Section 3 the double differential cross sections in $Q^{2}$ and $x$ were also measured. The double-differential cross section for inclusive beauty production in DIS can be expressed as:

$$
\frac{d \sigma^{b \bar{b}}}{d Q^{2} d x}=\frac{2 \pi \alpha^{2}}{x Q^{4}}\left(\left[1+(1-y)^{2}\right] F_{2}^{b \bar{b}}\left(Q^{2}, x\right)-y^{2} F_{L}^{b \bar{b}}\left(Q^{2}, x\right)\right)
$$



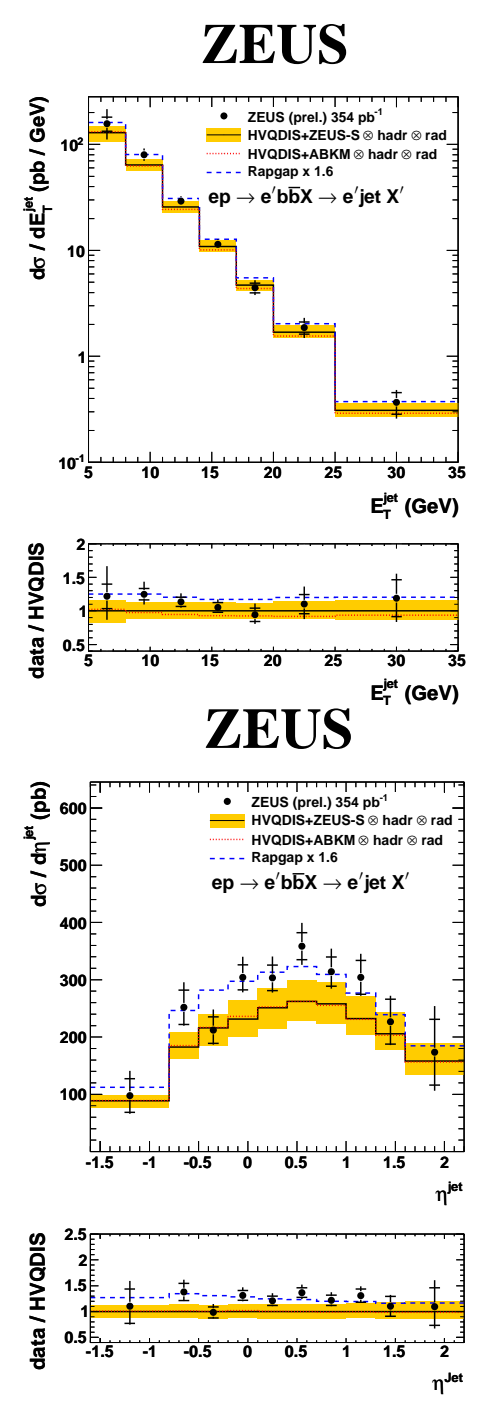

Figure 4: Measured differential cross sections as a function of $E_{\mathrm{T}}^{\mathrm{jet}}, \eta^{\text {jet }}$.

\section{HERA}

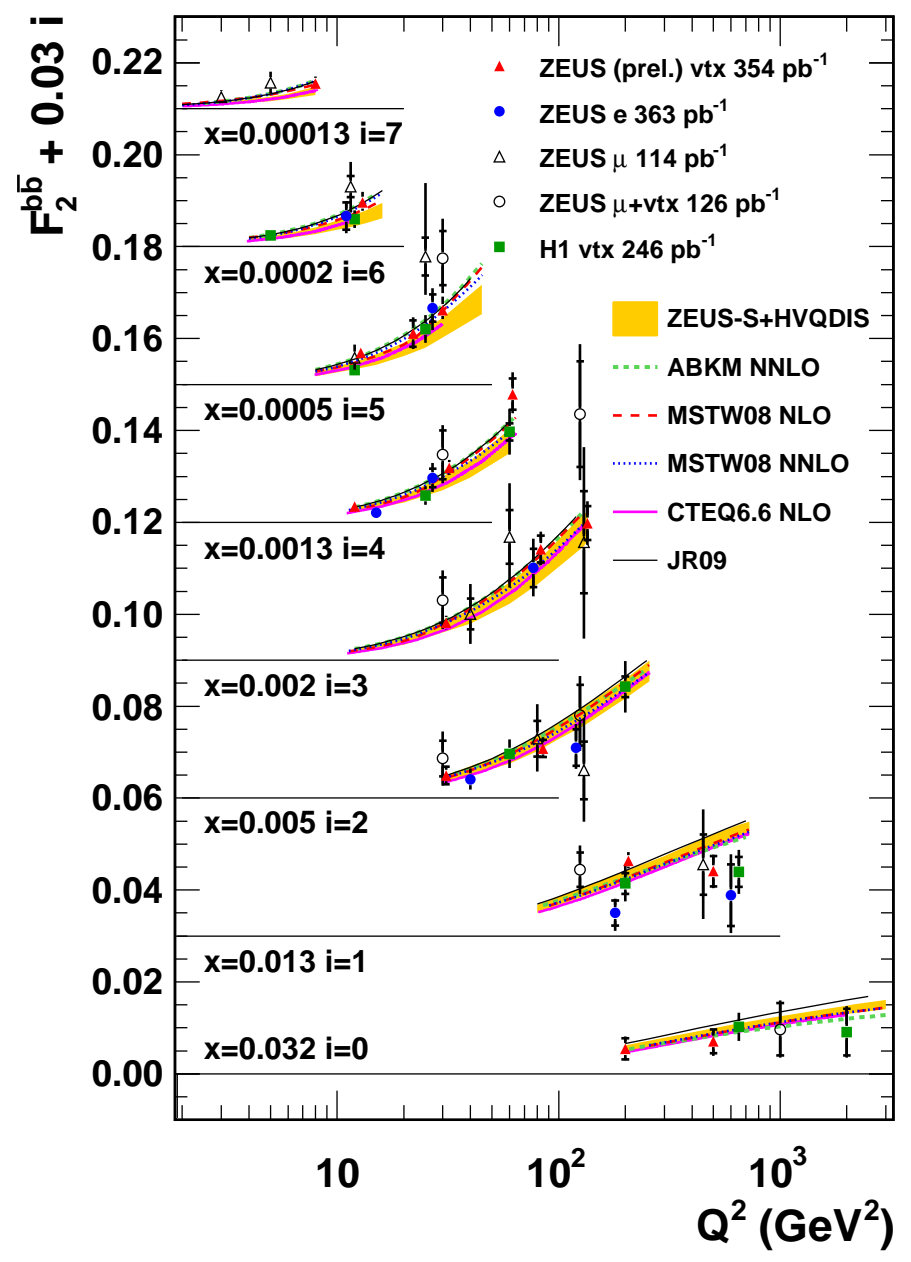

Figure 5: $F_{2}^{b \bar{b}}$ as a function of $Q^{2}$ at the fixed values of $x$. Results from the jet $+\mu$ (open triangles) and secondary vertex (red triangles) analyses are compared to the previous measurements by ZEUS and H1 (various symbols) and different predictions (lines and bands).

To extract $F_{2}^{b \bar{b}}$ from the measured visible cross sections in bins of $Q^{2}$ and $x$, an extrapolation to the full phase space was performed using NLO QCD calculations.

The results of the extrapolation are shown in Fig. 5. The new measurements agree with other ZEUS and $\mathrm{H} 1$ measurements [⿰] $]$ ] as well as with various theoretical predictions. The measurement with the secondary vertices is the most precise $F_{2}^{b \bar{b}}$ measurement from ZEUS.

\section{Conclusions}

Cross sections of beauty production in DIS were measured in events with muons and displaced secondary vertices. The measurements are well described by theoretical calculations. The beauty 
contribution to the structure function $F_{2}$ was found to be in agreement with previous H1 and ZEUS measurements as well as with various predictions.

\section{References}

[1] ZEUS Coll., H. Abramowicz et al., Eur. Phys. J. C 69 (2010) 347 [hep-ex/10 05. 3396].

[2] ZEUS Coll., ZEUS-prel-10-004.

[3] ZEUS Coll., H. Abramowicz et al., Eur. Phys. J. C 71 (2011) 1573;

ZEUS Coll., S. Chekanov et al., Eur. Phys. J. C 65 (2010) 65;

H1 Coll., F. Aaron et al., Eur. Phys. J. C 65 (2010) 89. 